\title{
ORIGINAL
}

\section{VIOLENCIA DE PAREJA EN JÓVENES DE 15 A 16 AÑOS DE LA COMUNIDAD DE MADRID}

\section{Myrian Pichiule Castañeda (1), Ana María Gandarillas Grande (1), Lucía Díez-Gañán (1), Michela Sonego (2) y María Ascensión Ordobás Gavín (1).}

(1) Servicio de Epidemiología. Subdirección de Promoción de la Salud y Prevención. Dirección General de Atención Primaria. Servicio Madrileño de Salud.

(2) Institute for Maternal and Child Health - Istituto di Ricovero e Cura a Carattere Scientifico "Burlo Garofolo". Trieste. Italia

Sin conflicto de intereses

\section{RESUMEN}

Fundamentos: La violencia de pareja en jóvenes es un importante problema de salud pública por su magnitud y repercusiones. El objetivo fue estimar su prevalencia según sexo y su asociación con factores sociodemográficos y conductas de riesgo para la salud.

Métodos: Se utilizó el Sistema de Vigilancia de Factores de Riesgo de Enfermedades No Transmisibles en población juvenil (SIVFRENT-J) 2011-2012 en una muestra representativa de estudiantes de $4^{\circ}$ curso de la Enseñanza Secundaria Obligatoria ( 15 a 16 años) de la Comunidad de Madrid. Se preguntó por violencia física (VF) y violencia sexual (VS). Se calculó la prevalencia con intervalos de confianza al 95\%(IC95\%). La asociación entre violencia física y/o sexual (VFS) y conductas de riesgo para la salud se analizó con la razón de prevalencia (RP IC95\%) mediante modelos lineales generalizados con vínculo logarítmico y familia binomial.

Resultados: En 1.713 chicas y 1.664 chicos con pareja la prevalencia de VF fue respectivamente del 2,9\% (IC95\%:2,0-3,9) y del 4,0\% (IC95\%:2,9$5,0)(\mathrm{p}>0,05)$, la de VS fue del 5,3\% (IC95\%:4,1-6,5) y $2,4 \%$ (IC95\%:1,6$3,2)(\mathrm{p}<0,001)$ y la de VFS $7,1 \%$ (IC95\%:5,6-8,6) y $5,1 \%($ IC $95 \%: 4,0-6,3)$ $(\mathrm{p}<0,05)$. En chicas, en los modelos explicativos, la VFS se asoció con desorden alimentario: RP:1,74 (IC95\%:1,14-2,66), conducta sexual de riesgo (CSR): RP:1,67 (IC95\%:1,11-2,50), consumo excesivo de alcohol, RP:1,57 (IC95\%:1,06-2,34) y mala salud percibida, RP:1,67 (IC95\%:1,08-2,57). En chicos, la VFS se asoció con ser extranjero: RP:2,05 (IC95\%:1,32-3,18), desorden alimentario: RP:2,79 (IC95\%:1,58-4,92), CSR: RP:2,22 (IC95\%:1,343,66 ) y consumo de drogas: RP:2,14 (IC95\%:1,39-3,28).

Conclusiones: La VFS predomina en chicas y se asocia a diferentesconductas de riesgo según el sexo.

Palabras clave: Violencia. Adolescentes. Prevalencia. Vigilancia epidemiológica. Estilo de vida. Conducta alimentaria. Conductas sexuales. Alcohol. Drogas ilegales. Sistema de Vigilancia de factor de riesgo Conductual.

\section{Correspondencia}

Myrian Pichiule Castañeda

C/ San Martín de Porres $\mathrm{N}^{\circ} 6,1^{\mathrm{a}}$ planta

28035 Madrid

myrianpc@hotmail.com

\section{ABSTRACT}

\section{Young People Dating Violence Surveillance in Madrid, Spain}

Background: Dating violence in young adults is an important public health issue because of its magnitude and impact on health. The goal of this study is to determine the prevalence 12 months before the survey by sex and its association with socio-demographic and health risk behavior.

Methods: Data were drawn from the Non-communicable Disease Risk-Factor Surveillance System in young people (Spanish acronym, SIVFRENT-J), on a representative sample of students of the fourth-year of secondary education (15 to 16 years) in Madrid Region. The students were asked whether they had ever suffered any physical violence (PV) and/or sexual violence (SV) dating episodes. Prevalence with $95 \%$ confidence intervals (CI95\%) of the occurrence of such episodes were estimated; generalized linear models with binomial family and logarithmic link were used to estimate the association between physical and/or sexual violence (PSV) and health risk behavior through prevalence ratios (PR, C195\%).

Results: In 1713 dating girls and 1664 dating boys, the prevalence of PV was $2.9 \%$ (CI95\%:2.0-3.9) and 4.0\% (CI95\%:2.9-5.0) (p>0.05), SV 5.3\% $(\mathrm{CI} 95 \%: 4.1-6.5)$ and $2.4 \%(\mathrm{CI} 95 \%: 1.6-3.2)(\mathrm{p}<0.001)$ and PSV $7.1 \%$ (CI95\%:5.6-8.6) and 5.1\% (CI95\%:4.0-6.3) $(\mathrm{p}<0.05)$ in that order. In the girls explanatory model, PSV was associated with eating disorders PR:1.74 (CI95\%:1.14-2.66), sexual risk behavior (SRB), PR:1.67 (CI95\%:1.11-2.50), excessive alcohol consumption, PR:1.57 (CI95\%:1.06-2.34) and worse perceived health, PR:1.67 (CI95\%:1.08-2.57). In boys, PSV was associated with having been born in a country other than Spain, PR:2.05 (CI95\%:1.32-3.18), eating disorders, PR:2.79 (CI95\%:1.58-4.92), SRB, PR:2.22 (CI95\%:1.343.66) and drug consumption, PR:2.14 (CI95\%:1.39-3.28).

Conclusion: VFS was higher in girls and was associated to others health risk factors with similar characteristics according to sex.

Keyword: Violence. Adolescent. Prevalence. Epidemiological Surveillance. Lifestyle. Eating behavior. Sexual behavior. Alcohol. Illicit drugs. Behavioral Risk Factor Surveillance System. 


\section{INTRODUCCIÓN}

La violencia de pareja en jóvenes definida como agresiones psicológicas, físicas y sexuales dentro de relaciones de pareja entre 13 y 19 años por un miembro de pareja heterosexual o del mismo sexo es altamente prevalente ${ }^{1}$ y ha emergido como un importante problema de salud pública. Existen diferencias importantes en las relaciones de pareja entre adultos y adolescentes, como la convivencia, la dependencia financiera y el control, que suelen ser más frecuentes en adultos, además los jóvenes tienen falta de experiencia para negociar dentro de sus relaciones de pareja ${ }^{2}$. Asimismo los adolescentes tienen dificultades para reconocer el abuso físico y sexual por parte de su pareja, ya que comportamientos de control y de celos pueden percibirse como signos de amor $^{3}$. Las jóvenes víctimas de violencia de pareja tienen mayor probabilidad de presentar estos problemas en su vida adulta ${ }^{4}$.

La prevalencia de violencia de pareja puede variar dependiendo de los criterios utilizados y de los países. Sin embargo las investigaciones disponibles muestran que es elevada. El Youth Risk Behavior Surveillance System (YRBSS), que analiza datos de 2011 en EEUU, muestra que el 9,3\% de chicas y el $9,5 \%$ de chicos fueron golpeados, recibieron bofetadas o fueron lastimados físicamente con intención por sus novias o novios y el 11,8\% de chicas y el $4,5 \%$ de chicos fueron alguna vez forzados a tener relaciones sexuales cuando ellos no querían ${ }^{5}$. En España, en un estudio realizado en 2013 en jóvenes entre 12 y 24 años, se encontró que el 3\% de las chicas habían recibido golpes alguna vez y el $6,1 \%$ fueron presionadas por sus parejas a actividades de tipo sexual en las que no querían participar ${ }^{6}$. En otras encuestas en las que se pregunta por agresiones sexuales en general se encontró que en Barcelona en 2012 el 2,5\% de chicas y el $1,7 \%$ de chicos de $4^{\circ}$ curso de la Enseñanza Secundaria Obligatoria (ESO) fueron forzados a mantener relaciones sexuales cuando ellos realmente no querían ${ }^{7} \mathrm{y}$ en Terrassa en 2010 al encuestar a jóvenes entre 14 y 18 años el $4,2 \%$ de chicas y el $1,5 \%$ de chicos declara que fueron forzados a mantener una relación sexual sin penetración ${ }^{8}$.

Los adolescentes expuestos a violencia de pareja sufren consecuencias en su salud mental y física, tanto a corto como a largo plazo. La violencia de pareja se ha asociado con depresión y bajos resultados escolares ${ }^{9}$, con el consumo de drogas, alcohol, tabaco ${ }^{10-14}$, con conductas sexuales de riesgo ${ }^{15-17}$, con desordenes alimentarios ${ }^{10,17,18}$ e incluso con ideas y tentativas suicidas ${ }^{19,20}$. En España es necesario investigar la violencia de pareja en jóvenes, tanto en chicas como en chicos.

El objetivo del estudio fue determinar la prevalencia de violencia de pareja física $y / 0$ sexual en la región de Madrid y analizar su asociación con factores sociodemográficos y con las principales conductas y factores de riesgo para la salud.

\section{MATERIAL Y MÉTODOS}

Diseño, fuente de datos y población de estudio. Estudio transversal de prevalencia que analiza datos procedentes del Sistema de Vigilancia de Factores de Riesgo asociados a Enfermedades No Transmisibles en población juvenil (SIVFRENT-J) que se realiza anualmente en una muestra representativa de la población escolarizada de $4^{\circ}$ curso de la ESO de la Comunidad de Madrid (CM). Los sujetos incluidos en el estudio fueron los jóvenes escolarizados en $4^{\circ}$ de la ESO los cuales fueron encuestados en 2011 y 2012 y que refierieron haber tenido pareja durante los 12 meses anteriores a la encuesta. El tamaño muestral teórico estimado para cada año fue de 2.000 jóvenes. A este tamaño muestral le correspondía un error de muestreo absoluto de $2,2 \%$, bajo el supuesto de muestreo aleatorio simple, para una prevalencia del $50 \%$ y un nivel de confianza del $95 \%$. El muestreo se hizo por conglomerados bietápico con estratificación de unidades en primera etapa (centros escolares). La asignación muestral en cada estrato fue proporcional al número de alumnos de 15 
y 16 años matriculados. Los criterios de estratificación fueron la zona geográfica (Madrid y resto de municipios) y la titularidad de los centros escolares (públicos y concertados/privados). La selección de las unidades en la segunda etapa fue constante y aleatoria, muestreando dos aulas por centro escolar. La entrevista se basó en un cuestionario autoadministrado en el aula cuya duración media de cumplimentación fue de 45 minutos, siendo la participación de los alumnos voluntaria y anónima ${ }^{21,22}$. A partir de 2011, tomando como modelo el YRBSS, se incluyó un módulo para la vigilancia de la violencia de pareja física (VF) y sexual (VS), dirigido a jóvenes de ambos sexos. En 2011 y 2012 la información se obtuvo de 92 centros escolares y 178 aulas. La tasa de respuesta global (colegios y estudiantes) fue del $82,1 \%$. Inicialmente se obtuvo información de 4.098 sujetos. La tasa de respuesta a la pregunta de VF fue del $96,8 \%$ y a la pregunta de VS de $96,5 \%$ y a alguna de las dos preguntas $97,8 \%$. Se excluyó a $91(2,2 \%)$ sujetos que no respondieron a ninguna de las dos preguntas sobre violencia de pareja y a $630(15,4 \%)$ sujetos que no tuvieron pareja durante el último año, por lo que el número de sujetos incluidos en nuestro estudio fue de 3.377 $(82,4 \%)$.

Mediciones. La violencia de pareja se estudió a través de dos preguntas: En los últimos 12 meses ¿alguna vez el chico o la chica con el/la que estás o estabas saliendo te dio una bofetada, patadas o te lastimó físicamente de alguna manera? y ¿alguna vez el chico o la chica con el/la que estás o estabas saliendo te impuso conductas de tipo sexual que tú rechazabas? Se consideró que un/una joven fue víctima de violencia física y/o sexual (VFS) cuando respondió sí a cualquiera de las dos preguntas. Sólo cuando los jóvenes respondieron sí a las preguntas de violencia de pareja tenían la opción de identificar el sexo del agresor (sí, el chico con el que estoy o estaba saliendo o sí, la chica con la que estoy o estaba saliendo)
Las variables sociodemográficas estudiadas fueron edad, sexo, país de nacimiento (España o fuera de España), convivencia con ambos padres, el máximo nivel de estudios alcanzado por estos (universitarios, secundarios o primarios/menos de primarios) y su situación laboral (si trabajaban o no).

Las conductas y factores de riesgo para la salud analizados fueron: resultado escolar: promedio-alto (cuando respondieron que se encontraban entre los que sacaban las mejores notas, en el grupo medio-alto o en el grupo medio) y bajo (cuando respondieron que se encontraban en el grupo medio bajo o entre los que sacaban peores notas). Se clasificó a los sujetos según presentaran o no sobrepeso u obesidad siguiendo los criterios de Cole a partir del peso y la talla autoreferidos ${ }^{23}$. Percepción de la imagen corporal según percibieran o no estar algo delgados o muy delgados. Desorden alimentario si en los últimos 12 meses presentó alguno de los comportamientos alimentarios para perder peso o controlar la figura: estar sin comer 24 horas o más, vómitos provocados o uso de laxantes, diuréticos o píldoras adelgazantes.

Conducta sexual de riesgo (CSR), basándonos en los hallazgos de algunos autores que estudiaron las conductas sexuales de ries$\mathrm{go}^{12,15,17}$. Se construyó un indicador de presencia de alguno de los siguientes criterios: haber tenido relaciones sexuales antes de los 15 años, no haber usado preservativo en la última relación sexual o haber tenido más de tres parejas sexuales en los últimos 6 meses.

Se consideró consumo de tabaco cualquier consumo ocasional o diario independientemente de la cantidad.

El consumo de alcohol se categorizó en: "no bebedores" (no consumieron alcohol en los últimos 30 días), "bebedores sin consumo excesivo en una misma ocasión" (consumieron $\geq 1$ bebida durante los últimos 30 días, pero que no realizaban un consumo excesivo de alcohol en una misma ocasión) y "bebedores 
con consumo excesivo en una misma ocasión" (consumieron 6 o más unidades de alcohol en una misma ocasión: binge drinking). Consumo de drogas según hubieran consumido en los últimos 12 meses alguna de las siguientes sustancias: tranquilizantes, marihuana, cocaína, heroína, anfetaminas, éxtasis, alucinógenos o sustancias volátiles.

Para la salud percibida las respuestas se agruparon en dos categorías, buena/muy buena o regular/mala/muy mala.

Análisis estadístico. Se estimó la prevalencia de VF, VS y VFS en los últimos 12 meses anteriores a la encuesta y su intervalo de confianza al 95\% (IC95\%) ajustando por el efecto de diseño muestral. Mediante modelos lineales generalizados con vínculo logarítmico y familia binomial se calcularon las razones de prevalencia (RP) crudas para el total de los jóvenes y según sexo y ajustadas por todas las variables estudiadas. Finalmente se construyeron modelos explicativos de VFS para el total de jóvenes y según sexo. Partiendo de un modelo saturado que incluía variables de interés y las que se asociaron a VFS con una $p \leq 0,20$, se fueron eliminando progresivamente las variables que no contribuían significativamente al modelo hasta obtener un modelo final.

Se utilizaron los módulos Survey Data y "glm" de STATA versión 10 (StataCorp., College Station, 2007) considerando en las comparaciones estadísticamente significativo un valor de p menor o igual a 0,05 .

\section{RESULTADOS}

Se analizó la información de 3.377 jóvenes con pareja en el último año. Las características sociodemográficas de los sujetos según sexo se presentan en la tabla 1. El test de significación estadística de comparación de proporciones indica que las características de chicas y chicos fueron similares.

La prevalencia de violencia 12 meses antes de la encuesta según sexo se presenta en la tabla 2, la prevalencia de VS y VFS fue significativa-

Tabla 1

Características sociodemográficas de los jóvenes con pareja en el último año según sexo

\begin{tabular}{|c|c|c|c|c|c|c|c|}
\hline \multirow{2}{*}{\multicolumn{2}{|c|}{ Variables }} & \multicolumn{2}{|c|}{ Chicas } & \multicolumn{2}{|c|}{ Chicos } & \multicolumn{2}{|c|}{ Total } \\
\hline & & $\mathrm{n}$ & $\%$ & $\mathbf{n}$ & $\%$ & $\mathbf{n}$ & $\%$ \\
\hline \multicolumn{2}{|l|}{ General } & 1.713 & 50,7 & 1.664 & 49,3 & 3.377 & 100 \\
\hline \multirow{3}{*}{ Edad } & 15 años & 626 & 36,7 & 572 & 34,7 & 1.198 & 35,7 \\
\hline & 16 años & 713 & 41,8 & 670 & 40,7 & 1.383 & 41,2 \\
\hline & $\geq 17$ años & 367 & 21,5 & 406 & 24,6 & 773 & 23,1 \\
\hline \multirow{2}{*}{ País de nacimiento } & España & 1.357 & 79,4 & 1.364 & 82,3 & 2.721 & 80,8 \\
\hline & Otro & 353 & 20,6 & 294 & 17,7 & 647 & 19,2 \\
\hline \multirow{2}{*}{ Convivencia con ambos padres } & Sí & 1.365 & 79,8 & 1.336 & 80,4 & 2.701 & 80,1 \\
\hline & No & 345 & 20,2 & 325 & 19,6 & 670 & 19,9 \\
\hline \multirow{2}{*}{ Trabaja el padre } & Sí & 1.422 & 86,1 & 1.437 & 88,7 & 2.859 & 87,4 \\
\hline & No & 230 & 13,9 & 184 & 11,3 & 414 & 12,6 \\
\hline \multirow{2}{*}{ Trabaja la madre } & Sí & 1.218 & 71,6 & 1.182 & 71,6 & 2.400 & 71,6 \\
\hline & No & 484 & 28,4 & 468 & 28,4 & 952 & 28,4 \\
\hline \multirow{3}{*}{ Estudios del padre } & Universitarios & 504 & 33,3 & 542 & 36,8 & 1.046 & 35,0 \\
\hline & Secundarios & 479 & 31,6 & 473 & 32,1 & 952 & 31,9 \\
\hline & Primarios o menos & 531 & 35,1 & 457 & 31,1 & 988 & 33,1 \\
\hline \multirow{3}{*}{ Estudios de la madre } & Universitarios & 541 & 33,9 & 550 & 36,2 & 1.091 & 35,0 \\
\hline & Secundarios & 514 & 32,2 & 509 & 33,5 & 1.023 & 32,8 \\
\hline & Primarios o menos & 541 & 33,9 & 460 & 30,3 & 1.001 & 32,1 \\
\hline
\end{tabular}


Tabla 2

Prevalencia de violencia de pareja física (VF), sexual (VS) y físico-sexual (VFS) según sexo

\begin{tabular}{|c|c|c|c|c|c|c|c|c|c|c|c|c|c|}
\hline \multirow{2}{*}{$\begin{array}{c}\text { Tipo de } \\
\text { violencia }\end{array}$} & \multicolumn{4}{|c|}{ Chicas } & \multicolumn{4}{|c|}{ Chicos } & \multirow{2}{*}{$\mathbf{p}^{*}$} & \multicolumn{4}{|c|}{ Total } \\
\hline & $\mathrm{n}$ & $\mathrm{C}$ & $\%$ & IC95\% & $\mathrm{n}$ & $\mathrm{C}$ & $\%$ & IC95\% & & $\mathrm{n}$ & $\mathrm{C}$ & $\%$ & IC95\% \\
\hline VF & 1.672 & 49 & 2,9 & $2,0-3,9$ & 1.638 & 65 & 4,0 & $2,9-5,0$ & 0,1454 & 3.310 & 114 & 3,4 & $2,8-4,1$ \\
\hline VS & 1.678 & 89 & 5,3 & $4,1-6,5$ & 1.638 & 39 & 2,4 & $1,6-3,2$ & 0,0001 & 3.316 & 128 & 3,9 & $3,1-4,6$ \\
\hline VFS & 1.713 & 122 & 7,1 & $5,6-8,6$ & 1.664 & 85 & 5,1 & $4,0-6,3$ & 0,0329 & 3.377 & 207 & 6,1 & $5,2-7,1$ \\
\hline
\end{tabular}

${ }^{*}$ Test de comparación de los tipos de violencia según sexo. n: Sujetos analizados C: víctimas de violencia

mente mayor en chicas. Se identificaron 207 casos de VFS de los cuales el 4,8\% ocurrieron entre parejas del mismo sexo.

La prevalencia de VFS según las características sociodemográficas, conductas y factores de riesgo para la salud se muestran en las tablas $3 \mathrm{y}$ 4. En chicas la prevalencia de VFS fue signifi- cativamente mayor entre aquellas con: desorden alimentario 12,9\% (IC95\%:8,9-16,8), CSR 12,5\% (IC95\%:8,2-16,9), consumo de tabaco 10,1\% (IC95\%:7,0-13,2), consumo no excesivo de alcohol 7,8\% (IC95\%:5,1-10,5) y consumo excesivo 9,0\% (IC95\%:6,7-11,3), consumo de drogas $10,4 \%$ (IC95\%:6,9-14,0) y con mala salud percibida 12,4\% (IC95\%:7,4-17,5). En

Tabla 3

Prevalencia de VFS según las características sociodemográficas

\begin{tabular}{|c|c|c|c|c|c|c|c|c|c|c|c|c|}
\hline \multirow{2}{*}{$\begin{array}{c}\text { Características } \\
\text { Sociodemográficas }\end{array}$} & \multicolumn{4}{|c|}{ Chicas } & \multicolumn{4}{|c|}{ Chicos } & \multicolumn{4}{|c|}{ Total } \\
\hline & $\mathbf{n}$ & $\mathbf{C}$ & $\%$ & IC95\% & $\mathbf{n}$ & $\mathbf{C}$ & $\%$ & IC95\% & $\mathbf{n}$ & $\mathbf{C}$ & $\%$ & IC95\% \\
\hline Total & 1.713 & 122 & 7,1 & $5,6-8,6$ & 1.664 & 85 & 5,1 & $4,0-6,3$ & 3.377 & 207 & 6,1 & $5,2-7,1$ \\
\hline \multicolumn{13}{|l|}{ Edad } \\
\hline 15 & 626 & 48 & 7,7 & $5,3-10,0$ & 572 & 21 & 3,7 & $2,1-5,2$ & 1.198 & 69 & 5,8 & $4,3-7,2$ \\
\hline 16 & 713 & 50 & 7,0 & $5,2-8,8$ & 670 & 32 & 4,8 & $3,0-6,5$ & 1.383 & 82 & 5,9 & $4,6-7,2$ \\
\hline$>=17$ & 367 & 24 & 6,5 & $3,8-9,3$ & 406 & 31 & 7,6 & $5,3-10,0$ & 773 & 55 & 7,1 & $5,1-9,1$ \\
\hline \multicolumn{13}{|l|}{ País nacimiento } \\
\hline España & 1.357 & 91 & 6,7 & $5,2-8,2$ & 1.364 & 56 & 4,1 & $3,1-5,1$ & 2.721 & 147 & 5,4 & $4,5-6,3$ \\
\hline Otro país & 353 & 30 & 8,5 & $5,3-11,7$ & 294 & 28 & 9,5 & $6,2-12,8$ & 647 & 58 & 9,0 & $6,8-11,1$ \\
\hline \multicolumn{13}{|c|}{ Convivencia con los dos padres } \\
\hline $\mathrm{Si}$ & 1.365 & 90 & 6,6 & $5,0-8,2$ & 1.336 & 70 & 5,2 & $4,0-6,5$ & 2.701 & 160 & 5,9 & $4,9-7,0$ \\
\hline No & 345 & 32 & 9,3 & $5,9-12,6$ & 325 & 15 & 4,6 & $2,1-7,1$ & 670 & 47 & 7,0 & $4,8-9,2$ \\
\hline \multicolumn{13}{|l|}{ Trabaja el padre } \\
\hline Sí & 1.422 & 98 & 6,9 & $5,4-8,4$ & 1.437 & 72 & 5,0 & $3,8-6,2$ & 2.859 & 170 & 5,9 & $5,0-6,9$ \\
\hline No & 230 & 19 & 8,3 & $4,3-12,2$ & 184 & 9 & 4,9 & $1,8-7,9$ & 414 & 28 & 6,7 & $4,1-9,4$ \\
\hline \multicolumn{13}{|l|}{ Trabaja la madre } \\
\hline Sí & 1.218 & 86 & 7,1 & $5,4-8,7$ & 1.182 & 51 & 4,3 & $3,1-5,5$ & 2.400 & 137 & 5,7 & $4,8-6,7$ \\
\hline No & 484 & 34 & 7,0 & $4,6-9,5$ & 468 & 34 & 7,3 & $4,6-9,9$ & 952 & 68 & 7,1 & $5,2-9,1$ \\
\hline \multicolumn{13}{|l|}{ Estudios del padre } \\
\hline Universitarios & 504 & 39 & 7,7 & $5,1-10,4$ & 542 & 26 & 4,8 & $2,9-6,7$ & 1.046 & 65 & 6,2 & $4,6-7,8$ \\
\hline Secundarios & 479 & 34 & 7,1 & $4,5-9,7$ & 473 & 23 & 4,9 & $2,7-7,0$ & 952 & 57 & 6,0 & $4,3-7,7$ \\
\hline Primarios o menos & 531 & 37 & 7,0 & $4,5-9,4$ & 457 & 27 & 5,9 & $3,7-8,1$ & 988 & 64 & 6,5 & $4,7-8,3$ \\
\hline \multicolumn{13}{|l|}{ Estudios de la madre } \\
\hline Universitarios & 541 & 38 & 7,0 & $4,4-9,6$ & 550 & 29 & 5,3 & $3,4-7,2$ & 1.091 & 67 & 6,1 & $4,6-7,7$ \\
\hline Secundarios & 514 & 39 & 7,6 & $5,0-10,2$ & 509 & 23 & 4,5 & $2,8-6,3$ & 1.023 & 62 & 6,1 & $4,5-7,6$ \\
\hline Primarios o menos & 541 & 38 & 7,0 & $4,7-9,3$ & 460 & 26 & 5,7 & $3,6-7,7$ & 1.001 & 64 & 6,4 & $4,8-8,0$ \\
\hline
\end{tabular}

$\mathrm{n}$ : Sujetos analizados C: víctimas de violencia 
Tabla 4

Prevalencia de VFS según las conductas y factores de riesgo para la salud

\begin{tabular}{|c|c|c|c|c|c|c|c|c|c|c|c|c|}
\hline \multirow{2}{*}{ FR } & \multicolumn{4}{|c|}{ Chicas } & \multicolumn{4}{|c|}{ Chicos } & \multicolumn{4}{|c|}{ Total } \\
\hline & $\mathbf{n}$ & $\mathbf{C}$ & $\%$ & IC95\% & $\mathbf{n}$ & $\mathbf{C}$ & $\%$ & IC95\% & $\mathbf{n}$ & $\mathrm{C}$ & $\%$ & IC95\% \\
\hline \multicolumn{13}{|l|}{ Resultado escolar } \\
\hline Promedio/alto & 1.379 & 91 & 6,6 & $4,9-8,3)$ & 1.240 & 53 & 4,3 & $3,0-5,5$ & 2.619 & 144 & 5,5 & $4,5-6,5$ \\
\hline Bajo & 331 & 31 & 9,4 & $5,9-12,8)$ & 418 & 31 & 7,4 & $4,8-10,0$ & 749 & 62 & 8,3 & $6,0-10,5$ \\
\hline \multicolumn{13}{|l|}{ Sobrepeso/Obesidad IMC } \\
\hline No & 1.480 & 111 & 7,5 & $5,8-9,2$ & 1.289 & 69 & 5,4 & $4,0-6,7$ & 2.769 & 180 & 6,5 & $5,4-7,6$ \\
\hline Sí & 167 & 10 & 6,0 & $2,5-9,5$ & 314 & 11 & 3,5 & $1,4-5,6$ & 481 & 21 & 4,4 & $2,6-6,1$ \\
\hline \multicolumn{13}{|l|}{ Delgadez percibida } \\
\hline No & 1.518 & 104 & 6,9 & $5,2-8,5$ & 1.296 & 61 & 4,7 & $3,5-5,9$ & 2.814 & 165 & 5,9 & $4,8-6,9$ \\
\hline Sí & 190 & 18 & 9,5 & $5,6-13,3$ & 354 & 23 & 6,5 & $3,8-9,2$ & 544 & 41 & 7,5 & $5,4-9,7$ \\
\hline \multicolumn{13}{|l|}{ Desorden alimentario } \\
\hline No & 1.402 & 83 & 5,9 & $4,4-7,4$ & 1.573 & 72 & 4,6 & $3,4-5,7$ & 2.975 & 155 & 5,2 & $4,3-6,1$ \\
\hline Sí & 303 & 39 & 12,9 & $8,9-16,8$ & 79 & 12 & 15,2 & $7,7-23,3$ & 382 & 51 & 13,4 & $9,7-17,0$ \\
\hline \multicolumn{13}{|l|}{ Conducta sexual de riesgo } \\
\hline No & 1.446 & 88 & 6,1 & $4,5-7,6$ & 1.323 & 46 & 3,5 & $2,5-4,4$ & 2.769 & 134 & 4,8 & $4,0-5,7$ \\
\hline Sí & 255 & 32 & 12,5 & $8,2-16,9$ & 327 & 38 & 11,6 & $7,5-15,7$ & 581 & 70 & 12,0 & $9,2-14,8$ \\
\hline \multicolumn{13}{|l|}{ Consumo actual de tabaco } \\
\hline No & 1.185 & 67 & 5,7 & $4,0-7,3$ & 1.241 & 52 & 4,2 & $3,1-5,3$ & 2.426 & 119 & 4,9 & $4,0-5,8$ \\
\hline Sí & 525 & 53 & 10,1 & $7,0-13,2$ & 420 & 33 & 7,9 & $5,2-10,5$ & 945 & 86 & 9,1 & $7,0-11,2$ \\
\hline \multicolumn{13}{|l|}{ Consumo de alcohol } \\
\hline No bebedor & 630 & 31 & 4,9 & $3,2-6,6$ & 698 & 21 & 3,0 & $1,5-4,5$ & 1.328 & 52 & 3,9 & $2,8-5,0$ \\
\hline Bebedor sin consumo excesivo & 513 & 40 & 7,8 & $5,1-10,5$ & 430 & 26 & 6,0 & $3,8-8,3$ & 943 & 66 & 7,0 & $5,3-8,7$ \\
\hline Bebedor con consumo excesivo & 566 & 51 & 9,0 & $6,7-11,3$ & 529 & 37 & 7,0 & $4,8-9,2$ & 1.095 & 88 & 8,0 & $6,4-9,7$ \\
\hline \multicolumn{13}{|l|}{ Consumo de drogas } \\
\hline Nunca/alguna vez & 1.404 & 91 & 6,5 & $4,9-8,0$ & 1.272 & 48 & 3,8 & $2,7-4,8$ & 2.676 & 139 & 5,2 & $4,3-6,1$ \\
\hline En los últimos 12 meses & 278 & 29 & 10,4 & $6,9-14,0$ & 353 & 36 & 10,2 & $6,6-13,8$ & 631 & 65 & 10,3 & $7,5-13,1$ \\
\hline \multicolumn{13}{|l|}{ Salud percibida } \\
\hline Buena/Muy Buena & 1.509 & 96 & 6,4 & $4,9-7,8$ & 1.539 & 73 & 4,7 & $3,6-5,9$ & 3.048 & 169 & 5,5 & $4,6-6,5$ \\
\hline No Buena & 201 & 25 & 12,4 & $7,4-17,5$ & 122 & 12 & 9,8 & $5,2-14,4$ & 323 & 37 & 11,5 & $8,0-15,0$ \\
\hline
\end{tabular}

n: Sujetos analizados C: víctimas de violencia

chicos la VFS fue más alta con diferencias significativas en: $\geq 17$ años 7,6\% (IC95\%:5,310,0 ), nacidos fuera de España 9,5\% (IC95\%: $6,2-12,8)$, con madres sin trabajo $7,3 \%$ (IC95\%:4,6-9,9), con bajos resultados escolares 7,4\% (IC95\%:4,8-10,0), desorden alimentario $15,2 \%($ IC $95 \%: 7,7-23,3)$, CSR 11,6\% (IC95\%:7,5-15,7), consumo de tabaco 7,9\% (IC95\%:5,2-10,5), consumo no excesivo de alcohol 6,0\% (IC95\%:3,8-8,3) y consumo excesivo 7,0\% (IC95\%:4,8-9,2), consumo de drogas 10,2\% (IC95\%:6,6-13,8), y entre aquellos con mala salud percibida 9,8\% (IC95\%:5,2-14,4). En el total de los jóvenes se encontró una preva- lencia significativamente más alta de VFS en chicas 7,1\% (IC95\%:5,6-8,6), en mayores de 17 años 7,1\% (IC95\%:5,1-9,1), con bajos resultados escolares 8,3\% (IC95\%:6,0-10,5), desorden alimentario 13,4\% (IC95\%:9,7-17,0), CSR 12,0\% (IC95\%:9,2-14,8), consumo de tabaco 9,1\% (IC95\%:7,0-11,2), consumo no excesivo de alcohol 7,0\% (IC95\%:5,3-8,7) y consumo excesivo 8,0\% (IC95\%:6,4-9,7), consumo de drogas $10,3 \%$ (IC95\%:7,5-13,1) y con mala salud percibida 11,5\% (IC95\%:8,0-15,0).

En la tabla 5 se presentan las RP crudas y ajustadas por todas las variables estudiadas. La 
Tabla 5

Razón de prevalencia de VFS según características sociodemográficas y conductas y factores de riesgo para la salud

\begin{tabular}{|c|c|c|c|c|c|c|}
\hline & \multicolumn{2}{|c|}{ Chicas } & \multicolumn{2}{|c|}{ Chicos } & \multicolumn{2}{|c|}{ Total } \\
\hline & RPc (IC95\%) & $\begin{array}{c}\operatorname{RPa}(\mathrm{IC} 95 \%) \\
(\mathrm{n}=1.372)\end{array}$ & RPc(IC95\%) & $\begin{array}{c}\mathrm{RPa}(\mathrm{IC} 95 \%) \\
(\mathrm{n}=1.319)\end{array}$ & $\operatorname{RPc}(\mathrm{IC} 95 \%)$ & $\begin{array}{c}\mathrm{RPa}(\mathrm{IC} 95 \%) \\
(\mathrm{n}=2.691)\end{array}$ \\
\hline \multicolumn{7}{|l|}{ Características Sociodemográficas } \\
\hline \multicolumn{7}{|l|}{ Edad } \\
\hline $16^{\mathrm{a}}$ & $0,91(0,66-1,26)$ & $0,82(0,58-1,17)$ & $1,30(0,76-2,21)$ & $1,17(0,64-2,12)$ & $1,03(0,76-1,39)$ & $0,93(0,68-1,29)$ \\
\hline$>=17^{\mathrm{a}}$ & $0,85(0,51-1,44)$ & $0,56(0,30-1,04)^{+}$ & $2,08(1,24-3,49)^{* *}$ & $1,29(0,70-2,38)$ & $1,24(0,85-1,79)$ & $0,81(0,53-1,24)$ \\
\hline Extranjero $^{b}$ & $1,27(0,86-1,87)$ & $1,09(0,64-1,86)$ & $2,32(1,57-3,44)^{* *}$ & $2,71(1,64-4,49)^{* *}$ & $1,66(1,27-2,17)^{* *}$ & $1,58(1,08-2,33)^{*}$ \\
\hline No Conviven con ambos padres ${ }^{c}$ & $1,41(0,93-2,12)^{\dagger}$ & $1,16(0,69-1,95)$ & $0,88(0,49-1,60)$ & $0,57(0,27-1,18)^{\dagger}$ & $1,18(0,83-1,69)$ & $0,91(0,62-1,34)$ \\
\hline Padre no trabaja ${ }^{\mathrm{d}}$ & $1,20(0,73-1,98)$ & $1,21(0,65-2,27)$ & $0,98(0,51-1,87)$ & $0,56(0,22-1,46)$ & $1,14(0,75-1,72)$ & $0,96(0,55-1,65$ \\
\hline Madre no trabaja ${ }^{\mathrm{d}}$ & $0,99(0,69-1,43)$ & $1,11(0,72-1,71)$ & $1,68(1,07-2,65)^{*}$ & $1,81(1,01-3,24)^{*}$ & $1,25(0,94-1,66)^{\dagger}$ & $1,32(0,96-1,83)^{\ddagger}$ \\
\hline \multicolumn{7}{|l|}{ Estudios del padre } \\
\hline Secundarios ${ }^{\mathrm{e}}$ & $0,92(0,58-1,46)$ & $0,90(0,54-1,49)$ & $1,01(0,57-1,79)$ & $0,77(0,43-1,37)$ & $0,96(0,66-1,40)$ & $0,85(0,57-1,26)$ \\
\hline Primaria o menos ${ }^{\mathrm{e}}$ & $0,90(0,56-1,44)$ & $0,64(0,35-1,17)^{\dagger}$ & $1,23(0,71-2,15)$ & $1,01(0,53-1,93)$ & $1,04(0,71-1,53)$ & $0,78(0,49-1,23)$ \\
\hline \multicolumn{7}{|l|}{ Estudios de la madre } \\
\hline Secundarios ${ }^{\mathrm{e}}$ & $1,08(0,64-1,81)$ & $0,99(0,57-1,73)$ & $0,86(0,50-1,46)$ & $0,78(0,39-1,56)$ & $0,99(0,69-1,41)$ & $0,90(0,60-1,35)$ \\
\hline Primarios o menos ${ }^{\mathrm{e}}$ & $1,00(0,63-1,60)$ & $1,13(0,62-2,05)$ & $1,07(0,67-1,71)$ & $0,89(0,44-1,80)$ & $1,04(0,75-1,45)$ & $1,03(0,65-1,64)$ \\
\hline \multicolumn{7}{|c|}{ Conductas y factores de riesgo para la salud } \\
\hline Bajo Resultado escolar ${ }^{\mathrm{f}}$ & $1,42(0,91-2,21)^{\dagger}$ & $1,25(0,78-2,03)$ & $1,74(1,11-2,72)^{*}$ & $1,19(0,69-2,06)$ & $1,51(1,10-2,06)^{*}$ & $1,26(0,89-1,80)^{\dagger}$ \\
\hline Sobrepeso/Obesidad IMC ${ }^{f}$ & $0,80(0,44-1,47)$ & $0,76(0,37-1,55)$ & $0,65(0,33-1,29)$ & $0,51(0,21-1,20)^{\dagger}$ & $0,67(0,44-1,02)^{+}$ & $0,63(0,37-1,07)^{*}$ \\
\hline Delgadez percibida $^{\mathrm{f}}$ & $1,38(0,87-2,19)^{*}$ & $1,23(0,68-2,21)$ & $1,38(0,85-2,25)^{\ddagger}$ & $1,34(0,75-2,38)$ & $1,29(0,94-1,76)^{\ddagger}$ & $1,33(0,92-1,93)^{\dagger}$ \\
\hline 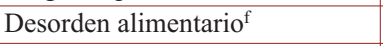 & $2,17(1,48-3,20)^{* *}$ & $1,73(1,12-2,70)^{*}$ & $3,31(1,82-6,07)^{* *}$ & $3,19(1,63-6,24)^{* *}$ & $2,56(1,86-3,52)^{* *}$ & $1,87(1,25-2,80)^{* *}$ \\
\hline Conducta sexual de riesgo $^{f}$ & $2,06(1,36-3,12)^{*}$ & $1,51(0,95-2,42)^{\ddagger}$ & $3,34(2,14-5,21)^{* *}$ & $2,04(1,25-3,33)^{* *}$ & $2,49(1,88-3,29)^{* *}$ & $1,72(1,27-2,34)^{* *}$ \\
\hline Consumo actual de tabaco ${ }^{\mathrm{f}}$ & $1,79(1,18-2,70)^{*}$ & $1,45(0,88-2,41)^{\dagger}$ & $1,88(1,26-2,80)^{*}$ & $1,20(0,72-1,98)$ & $1,86(1,40-2,45)^{* *}$ & $1,30(0,89-1,89)^{*}$ \\
\hline Bebedor sin consumo excesivog & $1,58(1,04-2,40)^{*}$ & $1,29(0,83-2,00)$ & $2,01(1,09-3,71)^{*}$ & $2,03(0,95-4,33)^{\dagger}$ & $1,79(1,27-2,52)^{*}$ & $1,52(1,06-2,20)^{*}$ \\
\hline Bebedor con consumo excesivo ${ }^{g}$ & $1,83(1,27-2,64)^{*}$ & $1,28(0,80-2,04)$ & $2,32(1,29-4,18)^{*}$ & $1,41(0,73-2,72)$ & $2,05(1,47-2,86)^{* *}$ & $1,38(0,91-2,09)^{\ddagger}$ \\
\hline Consumo de droga ${ }^{\mathrm{f}}$ & $1,61(1,10-2,36)^{*}$ & $1,09(0,59-2,00)$ & $2,70(1,73-4,23)^{* *}$ & $1,87(1,07-3,27)^{*}$ & $1,98(1,46-2,69)^{* *}$ & $1,40(0,93-2,13)^{\dagger}$ \\
\hline Mala salud percibida ${ }^{\mathrm{f}}$ & $1,96(1,28-2,99)^{*}$ & $1,57(0,90-2,72)^{\dagger}$ & $2,07(1,24-3,47)^{*}$ & $1,30(0,60-2,83)$ & $2,05(1,49-2,87)^{* *}$ & $1,48(0,99-2,29)^{*}$ \\
\hline
\end{tabular}

IC95\%: Intervalo de confianza al 95\%. RPc: Razón de prevalencia cruda. RPa: Razón de prevalencia ajustada por todas las variables de la tabla. Categoría de referencia $(\mathrm{RPc}=1 ; \mathrm{RPa}=1)$ : ${ }^{\mathrm{a}} 15$ años, ${ }^{\mathrm{b}}$ nacido en España, ${ }^{\mathrm{c}}$ convive con ambos padres, ${ }^{\mathrm{d}}$ trabajan, ${ }^{\mathrm{e}}$ universitarios, ${ }^{\mathrm{f}}$ no, g no bebedores. ${ }^{\dagger} \mathrm{p}<0,20 ;{ }^{\dagger} \mathrm{p}<0,10 ;{ }^{*} \mathrm{p}<0,05 ;{ }^{* *} \mathrm{p}<0,01$ 
Tabla 6

Modelo Explicativo de VFS

\begin{tabular}{|c|c|c|c|}
\hline & $\begin{array}{c}\text { Chicas } \\
\text { (Modelo A) } \\
(n=1.675) \\
\end{array}$ & $\begin{array}{c}\text { Chicos } \\
\text { (Modelo B) } \\
(n=1.564)\end{array}$ & $\begin{array}{c}\text { Total } \\
\text { (Modelo C) } \\
(\mathrm{n}=\mathbf{3 . 2 1 2}) \\
\end{array}$ \\
\hline \multicolumn{4}{|l|}{ Características sociodemográficas } \\
\hline Chicas $^{\mathrm{a}}$ & & & $1,26(0,91-1,74)^{\dagger}$ \\
\hline \multicolumn{4}{|l|}{ Edad } \\
\hline $16^{\mathrm{b}}$ & $0,87(0,64-1,18)$ & $1,13(0,67-1,90)$ & $0,94(0,70-1,26)$ \\
\hline$>=17^{b}$ & $0,65(0,38-1,10)^{\dagger}$ & $1,19(0,72-1,99)$ & $0,86(0,59-1,25)$ \\
\hline Extranjero $^{c}$ & $1,15(0,78-1,69)$ & $2,05(1,32-3,18)^{\dagger}$ & $1,46(1,10-1,95)^{*}$ \\
\hline Madre no trabaja ${ }^{\mathrm{d}}$ & & $1,67(1,00-2,80)^{\ddagger}$ & \\
\hline \multicolumn{4}{|c|}{ Conductas y factores de riesgo para la salud } \\
\hline Delgadez percibida $^{\mathrm{e}}$ & $1,55(0,93-2,57)^{\ddagger}$ & $1,39(0,81-2,36)$ & $1,45(1,04-2,02)^{*}$ \\
\hline Desorden alimentario $^{\mathrm{e}}$ & $1,74(1,14-2,66)^{*}$ & $2,79(1,58-4,92)^{* * *}$ & $1,87(1,28-2,73)^{* *}$ \\
\hline Conducta sexual de riesgo $^{\mathrm{e}}$ & $1,67(1,11-2,50)^{*}$ & $2,22(1,34-3,66)^{* *}$ & $1,85(1,40-2,45)^{* *}$ \\
\hline Bebedor sin consumo excesivo $^{f}$ & $1,45(0,95-2,20)^{\ddagger}$ & & $1,47(1,03-2,11)^{*}$ \\
\hline Bebedor con consumo excesivo $^{f}$ & $1,57(1,06-2,34)^{*}$ & & $1,39(0,94-2,05)^{\ddagger}$ \\
\hline Consumo de droga ${ }^{\mathrm{e}}$ & & $2,14(1,39-3,28)^{* * *}$ & $1,49(1,09-2,03)^{*}$ \\
\hline Mala salud percibida ${ }^{e}$ & $1,67(1,08-2,57)^{*}$ & & $1,52(1,09-2,11)^{*}$ \\
\hline
\end{tabular}

Categoría de referencia $(\mathrm{RP}=1)$ : a chicos, ${ }^{\mathrm{b}} 15$ años, ${ }^{\mathrm{c}}$ nacido en España, ${ }^{\mathrm{d}}$ madres que trabajan, ${ }^{\mathrm{e}}$ no, ${ }^{\mathrm{f}}$ no bebedores ${ }^{\dagger} \mathrm{p}<0,20 ;{ }^{\ddagger} \mathrm{p}<0,10 ;{ }^{*} \mathrm{p}<0,05 ;{ }^{* *} \mathrm{p}<0,01$

RP cruda de VFS de las chicas respecto a los chicos fue 1,39 (IC95\%:1,03-1,89) y la RP ajustada por todas las variables de la tabla fue de 1,23 (IC95\%:0,85-1,79) (información no mostrada en la tabla 4). En la tabla 6 se presentan los tres modelos explicativo de VFS. En el modelo A, realizado en chicas, encontramos que al ajustar por edad, país de nacimiento y delgadez percibida, la VFS se asoció con desorden alimentario (RP:1,74; IC 95\%:1, 14-2,66), CSR (RP: 1,67; IC95\%:1,11-2,50), consumo de alcohol (RP:1,57; IC95\%:1,06-2,34 en aquellas con consumo excesivo) y mala salud percibida (RP:1,67; IC95\%:1,08-2,57). En el modelo $\mathrm{B}$, en chicos, encontramos que al ajustar por edad, situación laboral de la madre y delgadez percibida la VFS se asocio con haber nacido fuera de España (RP:2,05; IC95\%:1,32-3,18), desorden alimentario (RP:2,79; IC95\%:1, 58-4,92), CSR (RP:2,22; IC95\%:1,34-3,66) y consumo de drogas (RP:2,14; IC95\%:1,39-3,28). En el modelo $\mathrm{C}$, para todos los jóvenes, encontramos que al ajustar por sexo y edad la VFS se asocio con haber nacido fuera de España
(RP:1,46; IC95\%:1,10-1,95), delgadez percibida (RP:1,45; IC95\%:1,04-2,02), desorden alimentario (RP:1,87; IC95\%:1,282,73), CSR (RP:1,85; IC95\%:1,40-2,45), consumo de alcohol (RP:1,47; IC95\%:1,032,11 en aquellos sin consumo excesivo), consumo de drogas (RP:1,49; IC95\%:1,09$2,03)$ y con mala salud percibida (RP:1,52; IC95\%:1,09-2,11).

\section{DISCUSIÓN}

La prevalencia de la VFS de pareja sufrida en jóvenes escolarizados de la Comunidad de Madrid muestra un predominio en las mujeres a expensas de la VS, mientras que la VF se da con una prevalencia similar en ambos sexos. Son varios los factores tanto sociodemográficos como hábitos de salud relacionados con la VFS, destacando por su mayor magnitud los desórdenes alimentarios y la conducta sexual de riesgo.

Los porcentajes de violencia física que encontramos en chicas son similares a los descritos en otro estudio realizado en Espa- 
ña que utiliza criterios similares ${ }^{6}$. Sin embargo, al utilizar otros instrumentos y ampliar el espacio temporal, estudios realizados en la Comunidad de Madrid encuentran porcentajes de violencia física más altos. Muñoz-Rivas utilizando la escala modificada de Conflict Tactics Scales (CTS) encuentra un porcentaje de violencia física de $37,4 \%$ en chicas y $31,3 \%$ en chicos con edades entre 16 y 20 años $^{24}$ y de $30,4 \%$ y $32,2 \%$ en jóvenes universitarios ${ }^{25}$. En otro estudio realizado en Salamanca en jóvenes de entre 15 y 19 años el porcentaje de víctimas de violencia física fue de $17,5 \%$ en chicas y $26,3 \%$ en $\operatorname{chicos}^{26}$.

En las comparaciones con estudios realizados fuera de nuestro país los porcentajes de violencia física de nuestro estudio son menores a los que describen los YRBSS ${ }^{5}$. En Canadá, utilizando una versión modificada del CTS, la prevalencia de violencia física en los últimos 5 años fue de $6,5 \%$ en chicas y 5,8\% en $\operatorname{chicos}^{27}$. En Tanzania y Sudáfrica donde utilizan la versión modificada del CTS-2, al estudiar a una población escolarizada de 10-18 años hallan porcentajes más altos de victimización y perpetración de violencia de pareja en $\operatorname{chicos}^{28}$.

En nuestro estudio encontramos porcentajes similares de violencia física en chicos y chicas, lo que podría explicarse debido a que existe un elevado porcentaje de agresiones mutuas en las relaciones de pareja en jóvenes $^{2}$. Y aunque la frecuencia de la violencia sea similar, diversas investigaciones sugieren que tanto la frecuencia de las agresiones, su gravedad y las consecuencias de las mismas son mayores en chicas que en $\operatorname{chicos}^{24,26}$. Cuando se estudian las motivaciones para usar la violencia, tras la cólera que suele ser la principal motivación en chicas y $\operatorname{chicos}^{2}$, las agresiones de las chicas ocurren en respuesta de la agresión recibida $^{24}$, mientras que en los chicos una de las motivaciones para usar la violencia es la necesidad de ejercer el control ${ }^{2}$.
En cuanto a la violencia sexual, al igual que otros estudios, los porcentajes más altos los hallamos en chicas y son similares a lo descrito por Díaz-Aguado ${ }^{6,29}$. En otras encuestas también se describen porcentajes superiores de violencia sexual en chicas $^{5,7,8}$.

Al igual que otros investigadores, con la finalidad de analizar la asociación entre violencia de pareja y las características sociodemográficas y conductas de riesgo para la salud agrupamos ambos tipos de violencia $(\mathrm{VFS})^{19}$. Jackson explica que existen algunos estudios en que utilizando el CTS encuentran mayores porcentajes de violencia física hacia chicos, sin embargo en estas encuestas no se tienen en cuenta la violencia sexual y la psicológica, que suelen ser más altas en chicas. En nuestro estudio la prevalencia de VFS fue superior en chicas comparado con la prevalencia en chicos, al igual que en otros como el de Ackard que encuentra un prevalencia de $8,6 \%$ en chicas y $6 \%$ en $\operatorname{chicos}^{18}$. Al analizar la prevalenica de violencia física y sexual en las relaciones de pareja en jóvenes es importante tener en cuenta las características peculiares de las relaciones a estas edades, en las que las dinámicas de poder son distintas, se encuentran aún en desarrollo las habilidades sociales y existe una gran influencia del grupo de amigos.

En cuanto a las características sociodemográficas para el total de jóvenes, encontramos asociación significativa entre VFS y haber nacido fuera de España; sin embargo, en el grupo de chicas no se encontró esta asociación, en ellas la VFS no se asoció a ninguna de las características sociodemográficas estudiadas. En los chicos la asociación entre VFS y la situación laboral de la madre es difícil de interpretar puesto que en este grupo se incluyen las amas de casa, las pensionistas y las que están en paro.

Al estudiar las conductas asociadas a violencia de pareja tanto en chicas como en chicos encontramos una fuerte asociación entre 
violencia y desórdenes alimentarios, lo que concuerda con los hallazgos de distintos estudios ${ }^{10,17,18,30}$. Hay otros estudios que describen asociación entre abuso sexual infantil y subsecuentes desórdenes alimentarios. Fischer encuentra que el contacto sexual no deseado se encuentra significativamente asociado con desórdenes alimentarios y este efecto persiste al ajustarlo por el abuso producido en la infancia ${ }^{31}$. Los desórdenes de la conducta alimentaria pueden constituir un intento de las víctimas de violencia de castigarse por el abuso, con el objetivo de disminuir las emociones desagradables conectadas con el abuso o con la finalidad de prevenir un posible abuso haciéndose a ellas poco atractivas ${ }^{18}$. Esta asociación entre VFS y desórdenes alimentarios se ha descrito de manera independiente tanto en chicas como en chicos ${ }^{16,32}$.

Al igual que en nuestro estudio existen otras investigaciones que han encontrado asociación entre la violencia de pareja en los últimos 12 meses y conductas sexuales de riesgo, como tener menor edad cuando se tienen relaciones sexuales, tener un elevado número de parejas sexuales y una menor probabilidad de utilizar condón en la última relación sexual ${ }^{12,15-17}$.

La evidencia sugiere que la edad de inicio de las relaciones sexuales está disminuyendo tanto en la Comunidad de Madrid como en otros países industrializados ${ }^{22,33,34}$, la edad temprana de inicio de relaciones sexuales no solo puede incrementar el riesgo de embarazos no deseados sino también las infecciones de transmisión sexual. Aunque el porcentaje de jóvenes que utilizaron condón en la última relación sexual es elevada en la Comunidad de Madrid, este porcentaje ha presentado un descenso especialmente en chicas si lo comparamos con lo que ocurría hace diez años ${ }^{22,33}$. Esta tendencia descendente también se aprecia en otras encuestas $^{7,8}$. Decker encontró asociación entre violencia de pareja física y sexual y mayor probabilidad de ser diagnosticado de infecciones de transmisión sexual, incluida la infección por virus de la inmunodeficiencia humana ${ }^{35}$.

Se encontró asociación independiente entre violencia de pareja y consumo de alcohol, drogas y tabaco, tanto en chicas como en chicos. Sin embargo en los modelos explicativos la violencia se asoció con el consumo de alcohol en chicas y con el consumo de drogas en chicos. El consumo de estas sustancias puede desencadenar el episodio de violencia ${ }^{14}$. Asimismo la violencia y los factores de riesgo como consumo de sustancias y comportamientos sexuales de riesgo actúan de manera bidireccional ${ }^{11,20}$.

El consumo frecuente y excesivo de alcohol se asocia con consecuencias adversas en la salud física, social y psicológica incluida violencia y relaciones sexuales no protegidas $^{36}$. Existen diversos estudios que encuentran asociación entre agresiones físicas y sexuales y consumo frecuente y excesivo de alcohol, incluso encuentran una relación dosis respuesta entre consumo excesivo de alcohol y otras conductas de riesgo $^{12-14,37}$.

La droga ilegal que más frecuentemente consumieron los jóvenes en la Comunidad de Madrid fue la marihuana. El consumo de cannabis alguna vez en la vida así como en los últimos 30 días en jóvenes españoles de 15 años es mayor si lo comparamos con la media a nivel europeo ${ }^{38}$. El consumo de esta sustancia se ha relacionado con externalización de problemas incluidos la exposición a riesgos y agresiones. La asociación entre experimentar violencia de pareja y consumo de drogas ha sido descrita en distintos estudios $9,11,13,18$. Es posible que estos jóvenes adopten estas conductas de riesgo como una forma temporal de escapar de los aspectos estresantes de la violencia. Los jóvenes que se involucran en conductas de riesgo podrían tender a asociarse con iguales con las mismas conductas ${ }^{14}$. 
La salud autorreportada es un indicador subjetivo de salud general. En adolescentes la salud percibida se asocia de manera independiente con indicadores médicos, sociales y personales ${ }^{39}$. La mala salud autopercibida se asoció con violencia de manera independiente en chicas y chicos y en los modelos explicativos esta asociación persiste en chicas. La violencia de pareja tiene un impacto negativo en la salud física y mental. Esto también es un indicador de que la actitud de las chicas frente a la violencia es distinta a la de los chicos, con independencia de la frecuencia de las agresiones, el grado de terror o el riesgo a una lesión física es más alto en chicas.

Algunas de las limitaciones del estudio han sido que la consideración de joven con pareja se realizó por exclusión, cuando el joven respondió que no había salido con nadie en los últimos 12 meses y se dejó que decidiese lo que considera salir con algún chico o chica, lo que finalmente consideramos tener pareja. Tampoco fue posible excluir del análisis las relaciones de pareja del mismo sexo, puesto que sólo se tuvo información del sexo de la pareja cuando el joven o la joven refirió ser víctima de algún tipo de violencia, en caso de que no fueran víctimas de violencia no se especificó el tipo de relación. Así del total de casos de VFS en jóvenes las que ocurren entre personas del mismo sexo representan un porcentaje menor, puesto que a nivel poblacional, este tipo de relaciones son menos frecuentes.

El problema se estudia en el marco de un sistema de vigilancia que abarca gran número de comportamientos relacionados con la salud, por lo que al ser el espacio limitado necesariamente debe basarse en pocas preguntas. Por la dificultad del tema no se ha estudiado la violencia psicológica, difícil de abordar fuera de una encuesta específica. Tampoco se han podido estudiar otros comportamientos de victimización, frecuencia y gravedad de la violencia. La comparación de los resultados debe hacerse con cautela y con estudios similares y puesto que el estudio parte de una encuesta transversal solo es posible encontrar asociaciones y no una relación causal.

Para vigilar las conductas de riesgo para la salud en adolescentes, es necesario obtener información autorreferida, cuya calidad puede verse comprometida por problemas de fiabilidad y validez. Estudios llevados a cabo en sistemas de información similares han obtenido una buena reproductibilidad para las preguntas de la esfera de violencia a diferencia de otras como el control de peso que muestran una menor repetibilidad ${ }^{40,41}$ En cuanto a la validez de preguntas relacionadas con violencia en jóvenes, dada la escases de investigaciones sobre el tema y la dificultad de verificar esta información por métodos objetivos teniendo en cuenta aspectos éticos y costoefectivos, al revisar la evidencia científica se han descrito dos tipos de factores que pueden afectar a la validez: cognitivos y del entorno ${ }^{42}$. En orden a controlar estos factores, en la elaboración de las preguntas se tuvo muy en cuenta su fácil interpretación. En cuanto a los factores externos, tanto la privacidad como el anonimato estuvieron garantizados en este estudio, por lo que pensamos que este tipo de validez no estará seriamente comprometida. Sería deseable realizar estudios de validación de este sistema de vigilancia en jóvenes tal como se ha realizado en adultos ${ }^{43}$.

En conclusión, alrededor de un 6\% de jóvenes sufren VFS, sobre todo las mujeres a causa de la violencia sexual. La prevalencia tanto en chicas como en chicos es menor a la que se describe en otras regiones según estudios con metodología similar. La vigilancia de violencia de pareja mediante el SIVFRENT-J permite aproximarse a la magnitud del problema y al ser un sistema anual y utilizar las mismas preguntas podría permitir monitorizar su evolución y generar información básica para planificar y evaluar 
intervenciones precoces en este colectivo con objeto de reducir las tasas de violencia. De los resultados de este estudio se desprende la necesidad de potenciar y reforzar la educación sexual en jóvenes, integrando la educación frente a la violencia de pareja en el marco general de trabajo de otros comportamientos de riesgo para la salud, con los que está asociada.

\section{AGRADECIMIENTOS}

Deseamos expresar nuestro agradecimiento a Belén Zorrilla, Iñaki Galán, Marisa Pires, Luisa Lasheras, Ana Anes y Jesús Sánchez por la colaboración en la elaboración de las preguntas de violencia de pareja incluidas en el sistema de vigilancia.

\section{BIBLIOGRAFÍA}

1. Cutter-Wilson E, Richmond T. Understanding teen dating violence: practical screening and intervention strategies for pediatric and adolescent healthcare providers. Curr Opin Pediatr. 2011;23(4):379-83.

2. Mudolford C, Giordano P. Teen dating violence: A closer look at adolescent romantic relationships. National Institute of Justice Journal. 2008;261:34-40.

3. Levy, B. Abusive teen dating relationship: An emerging issue for the 90s. Response to the Victimization of Women and Children. 1990;13(1):59.

4. Smith PH, White JW, Holland LJ. A longitudinal perspective on dating violence among adolescent and college-age women. Am J Public Health. 2003;93(7):1104-9.

5. Centers for Disease Control and Prevention. Youth Risk Behavior Surveillance-United States. MMWR. 2012;61(4).

6. Díaz-Aguado MJ, Martínez R, Martín J. Igualdad y prevención de violencia de género en la adolescencia. Madrid: Ministerio de Igualdad-Delegación de Gobierno para la Violencia de Género; 2013.

7. Pérez A, Garcia-Continente X i Grup col laborador enquesta FRESC 2012. Informe FRESC 2012: 25 anys d'enquestes a adolescents escolaritzats de Barcelona. Barcelona: Agència de Salut Pública de Barcelona, 2013.
8. Schiaffino A, Moncada A. Estudi EMCSAT 2010 Conductes de salut de la població adolescent escolaritzada de Terrassa,1993-2010. Terrassa: Ajuntament de Terrassa, 2011.

9. Banyard VL, Cross C. Consequences of teen dating violence: understanding intervening variables in ecological context. Violence Against Women. 2008;14(9):998-1013.

10. Ackard DM, Neumark-Sztainer D. Multiple sexual victimizations among adolescent boys and girls: prevalence and associations with eating behaviors and psychological health. J Child Sex Abus. 2003;12(1):17-37.

11. Brady SS, Tschann JM, Pasch LA, Flores E, Ozer EJ. Violence involvement, substance use, and sexual activity among Mexican-American and EuropeanAmerican adolescents. J Adolesc Health. 2008;43(3):285-95.

12. Coker AL, McKeown RE, Sanderson M, Davis $\mathrm{KE}$, Valois RF, Huebner ES. Severe dating violence and quality of life among South Carolina high school students. Am J Prev Med. 2000;19(4):220-7.

13. Miller JW, Naimi TS, Brewer RD, Jones SE. Binge drinking and associated health risk behaviors among high school students. Pediatrics. 2007;119(1):76-85.

14. Munoz-Rivas MJ, Gamez-Guadix M, Grana JL, Fernandez L. Violencia en el noviazgo y consumo de alcohol y drogas ilegales entre adolescentes y jóvenes españoles. Adicciones. 2010;22(2):125-33.

15. Alleyne B, Coleman-Cowger VH, Crown L, Gibbons MA, Vines LN. The effects of dating violence, substance use and risky sexual behavior among a diverse sample of Illinois youth. J Adolesc. 2011;34(1):11-8.

16. Bonomi AE, Anderson ML, Nemeth J, Rivara FP, Buettner C. History of dating violence and the association with late adolescent health. BMC Public Health. 2013;13:821.

17. Silverman JG, Raj A, Mucci LA, Hathaway JE. Dating violence against adolescent girls and associated substance use, unhealthy weight control, sexual risk behavior, pregnancy, and suicidality. JAMA. 2001;286(5):572-9.

18. Ackard DM, Neumark-Sztainer D. Date violence and date rape among adolescents: associations with disordered eating behaviors and psychological health. Child Abuse Negl. 2002;26(5):455-73. 
19. Ackard DM, Eisenberg ME, Neumark-Sztainer D. Long-term impact of adolescent dating violence on the behavioral and psychological health of male and female youth. J Pediatr. 2007;151(5):476-81.

20. Roberts TA, Klein JD, Fisher S. Longitudinal effect of intimate partner abuse on high-risk behavior among adolescents. Arch Pediatr Adolesc Med. 2003;157(9):875-81.

21. Díez-Gañán L. Hábitos de salud en la población juvenil de la Comunidad de Madrid, 2011. Resultados del Sistema de Vigilancia de Factores de Riesgo Asociados a Enfermedades no Transmisibles en población juvenil (SIVFRENT-J). Año 2011. Boletín Epidemiológico de la Comunidad de Madrid. Madrid: Consejería de Sanidad; 2012.

22. Díez-Gañán L. Hábitos de salud en la población juvenil de la Comunidad de Madrid, 2012. Resultados del Sistema de Vigilancia de Factores de Riesgo Asociados a Enfermedades no Transmisibles en población juvenil (SIVFRENT-J). Año 2012. Boletín Epidemiológico de la Comunidad de Madrid. Madrid: Consejería de Sanidad; 2013.

23. Cole TJ, Bellizzi MC, Flegal KM, Dietz WH. Establishing a standard definition for child overweight and obesity worldwide: international survey. BMJ. 2000;320(7244):1240-3.

24. Munoz-Rivas MJ, Grana JL, O'Leary KD, Gonzalez MP. Aggression in adolescent dating relationships: prevalence, justification, and health consequences. J Adolesc Health. 2007;40(4):298-304.

25. Munoz-Rivas MJ, Grana Gomez JL, O'Leary KD, Gonzalez LP. Physical and psychological aggression in dating relationships in Spanish university students. Psicothema. 2007;19(1):102-7.

26. Fernandez-Fuertes AA, Fuertes A. Physical and psychological aggression in dating relationships of Spanish adolescents: motives and consequences. Child Abuse Negl. 2010;34(3):183-91.

27. Ansara DL, Hindin MJ. Exploring gender differences in the patterns of intimate partner violence in Canada: a latent class approach. J Epidemiol Community Health. 2010;64(10):849-54.

28. Wubs AG, Aaro LE, Flisher AJ, Bastien S, Onya HE, Kaaya $\mathrm{S}$ et al. Dating violence among school students in Tanzania and South Africa: prevalence and socio-demographic variations. Scand J Public Health. 2009;37 Suppl 2:75-86.

29. Díaz-Aguado MJ, Martínez r, Martín J. Igualdad y prevención de violencia de género en la adolescencia. Madrid; 2010.
30. Paniagua-Repetto H, García-Calatayud S. Signos de alerta de trastornos alimentarios, de depresión, del aprendizaje y conductas violentas entre adolescentes de Cantabria. Rev Esp Salud Pública. 2003;77(3):411-22.

31. Fischer S, Stojek M, Hartzell E. Effects of multiple forms of childhood abuse and adult sexual assault on current eating disorder symptoms. Eat Behav. 2010;11(3):190-2.

32. Gault-Sherman M, Silver E, Sigfusdottir ID. Gender and the associated impairments of childhood sexual abuse: a national study of Icelandic youth. Soc Sci Med. 2009;69(10):1515-22.

33. Consejería de Sanidad y Servicios Sociales. Hábitos de salud en la población juvenil de la Comunidad de Madrid, 2002. Boletín Epidemiológico de la Comunidad de Madrid. Madrid. 2002.

34. Wellings K, Collumbien M, Slaymaker E, Singh S, Hodges Z, Patel D et al. Sexual behaviour in context: a global perspective. Lancet. 2006;368(9548):1706-28.

35. Decker MR, Silverman JG, Raj A. Dating violence and sexually transmitted disease/HIV testing and diagnosis among adolescent females. Pediatrics. 2005;116(2):e272-6.

36. Windle M. Alcohol use among adolescents and young adults. Alcohol Res Health. 2003;27(1):79-85.

37. Ackard DM, Neumark-Sztainer D, Hannan P. Dating violence among a nationally representative sample of adolescent girls and boys: associations with behavioral and mental health. J Gend Specif Med. 2003;6(3):39-48.

38. Currie $\mathrm{C}$ et al., eds. Social determinants of health and well-being among young people. Health Behaviour in School-aged Children (HBSC) study: International report from the 2009/2010 survey. Copenhagen, WHO regional Office for Europe, 2012 (Health Policy for children and Adolescents, No. 6).

39. Breidablik HJ, Meland E, Lydersen S. Self-rated health in adolescence: a multifactorial composite. Scand J Public Health. 2008;36(1):12-20.

40. Rosenbaum JE. Truth or consequences: the intertemporal consistency of adolescent self-report on the Youth Risk Behavior Survey. Am J Epidemiol. 2009;169(11):1388-97.

41. Brener ND, Kann L, Shanklin S Kinchen S, Eaton DK, Hawkins J, et al. Methodology of the Youth Risk Behavior Surveillance System--2013. MMWR Recomm Rep. 2013;62(RR-1):1-20. 
42. Brener ND, Billy JO, Grady WR. Assessment of factors affecting the validity of self-reported healthrisk behavior among adolescents: evidence from the scientific literature. J Adolesc Health. 2003;33(6):43657.

43. Galan I, Rodriguez-Artalejo F, Zorrilla B. Reproductibilidad de un cuestionario telefónico sobre factores de riesgo asociados al comportamiento y las prácticas preventivas. Gac Sanit. 2004;18(6):440-50. 